\title{
MMP13 mutation causes spondyloepimetaphyseal dysplasia, Missouri type $\left(\mathrm{SEMD}_{\mathrm{MO}}\right)$
}

\author{
Ann M. Kennedy, ${ }^{1}$ Masaki Inada, ${ }^{2}$ Stephen M. Krane, ${ }^{2}$ Paul T. Christie, ${ }^{1}$ Brian Harding, ${ }^{1}$ \\ Carlos López-Otín, ${ }^{3}$ Luis M. Sánchez, ${ }^{3}$ Anna A.J. Pannett, ${ }^{1}$ Andrew Dearlove, ${ }^{4}$ Claire Hartley, ${ }^{4}$ \\ Michael H. Byrne, ${ }^{2}$ Anita A.C. Reed, ${ }^{1}$ M. Andrew Nesbit, ${ }^{1}$ Michael P. Whyte, ${ }^{5}$ and Rajesh V. Thakker ${ }^{1}$

\begin{abstract}
${ }^{1}$ Academic Endocrine Unit, Nuffield Department of Clinical Medicine, University of Oxford, Oxford Centre for Diabetes, Endocrinology and Metabolism Diseases, Massachusetts General Hospital, Boston, Massachusetts, USA. ${ }^{3}$ Departamento de Bioquímica y Biología Molecular, Instituto Universitario de Oncologia, Universidad de Oviedo, Oviedo, Spain. ${ }^{4}$ Medical Research Council (MRC), Human Genome Mapping Project Resource Centre, Cambridge, United Kingdom. ${ }^{5}$ Center for Metabolic Bone Disease and Molecular Research, Shriners Hospitals for Children, St. Louis, Missouri, USA.
\end{abstract} \\ (OCDEM), Churchill Hospital, Oxford, United Kingdom. ${ }^{2}$ Department of Medicine, Harvard Medical School, and the Center for Immunology and Inflammatory
}

\begin{abstract}
MMPs, which degrade components of the ECM, have roles in embryonic development, tissue repair, cancer, arthritis, and cardiovascular disease. We show that a missense mutation of MMP13 causes the Missouri type of human spondyloepimetaphyseal dysplasia $\left(\mathrm{SEMD}_{\mathrm{MO}}\right)$, an autosomal dominant disorder characterized by defective growth and modeling of vertebrae and long bones. Genome-wide linkage analysis mapped $\mathrm{SEMD}_{\mathrm{MO}}$ to a 17-cM region on chromosome 11q14.3-23.2 that contains a cluster of 9 MMP genes. Among these, MMP13 represented the best candidate for $S \mathrm{SD}_{\mathrm{MO}}$, since it preferentially degrades collagen type II, abnormalities of which cause skeletal dysplasias that include Strudwick type SEMD. DNA sequence analysis revealed a missense mutation, F56S, that substituted an evolutionarily conserved phenylalanine residue for a serine in the proregion domain of MMP13. We predicted, by modeling MMP13 structure, that this F56S mutation would result in a hydrophobic cavity with misfolding, autoactivation, and degradation of mutant protein intracellularly. Expression of wild-type and mutant MMP13s in human embryonic kidney cells confirmed abnormal intracellular autoactivation and autodegradation of F56S MMP13 such that only enzymatically inactive, small fragments were secreted. Thus, the F56S mutation results in deficiency of MMP13, which leads to the human skeletal developmental anomaly of SEMD ${ }_{M O}$.
\end{abstract}

\section{Introduction}

Genetic disorders of bone (skeletal dysplasias) may have a variety of biochemical and molecular mechanisms, and the previous radiological classification, although useful for a diagnostic approach, is being supplemented by a molecular classification $(1,2)$. This is illustrated by the spondyloepimetaphyseal dysplasias (SEMDs), which are a heterogenous group of skeletal diseases featuring defective growth and modeling of the spine and long bones (1-5). The SEMDs often arise sporadically, but distinctive heritable forms with autosomal dominant, autosomal recessive, or X-linked transmission have been reported $(5,6)$. The pattern and severity of metaphyseal and epiphyseal involvement varies among and within affected SEMD kindreds, and some manifest additional features such as hypotrichosis or joint laxity (6-8). Biochemical markers of skeletal homeostasis are typically normal in patients with SEMDs and yield no clues about the etiologies $(1,2)$. However, radiological and histopathological studies have indicated that the SEMDs likely reflect fundamental disturbances in growth plate development $(5,9)$. Indeed, studies of kindreds have identified genetic defects in 3 autosomal SEMDs: the dominant Strudwick type,

Nonstandard abbreviations used: APMA, $p$-aminophenyl mercuric acetate; COL2A1, type II collagen (gene); HEK293 cell, human embryonic kidney 293 cell; IVT, in vitrotranslated/in vitro translation; LOD, $\log _{10}$ of odds; SED, spondyloepiphyseal dyspla-

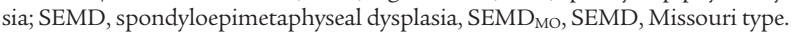
Conflict of interest: The authors have declared that no conflict of interest exists.

Citation for this article: J. Clin. Invest. 115:2832-2842 (2005).

doi:10.1172/JCI22900. the recessive Pakistani type, and recessive matrilin 3 deficiency $(3,4,10)$. Strudwick type SEMD is due to missense mutations of the type II collagen (COL2A1) gene, located on chromosome 12q1213 (3), and these result in abnormal posttranslational modification of the $\alpha-1$ (II) collagen chains (3). Pakistani type SEMD is due to a mutation of the ATP sulfurylase/APS kinase 2 gene (ATPSK2), located on chromosome 10q23-34, and this mutation likely impairs posttranslational sulfation of cartilage ECM, causing defects in maturation and growth plate function (4). The matrilin 3 gene is located on chromosome 2p24-25, and homozygosity for a missense mutation caused SEMD in a consanguineous Arab family (10). Matrilin 3 is one of a family of 4 oligomeric ECM proteins, and disruption of possible interactions with other proteins, such as collagen types II and IX, may destabilize the ECM structure (10). However, it is important to note that mutations in these genes may cause skeletal disorders other than the $\operatorname{SEMDs}(1,2)$. For example, COL2A1 mutations cause at least 8 type II collagenopathies (1-3), including spondyloepiphyseal dysplasia (SED) congenita, SED Namaqualand type, mild SED with premature onset arthrosis, Stickler dysplasia type I, spondyloperipheral dysplasia, Kniest dysplasia, hypochondrogenesis, and achondrogenesis II; and matrilin 3 mutations cause forms of multiple epiphyseal dysplasia (10). Mutations in these genes do not cause the autosomal dominant Missouri type of SEMD (SEMD MO $_{\text {) }}$ (11) (Figure 1).

$S_{\text {SEM }}$ MO, whose clinical and radiographic features were reported in 1993 (5), is characterized by moderate to severe metaphyseal changes, mild epiphyseal involvement, and pear-shaped vertebrae 


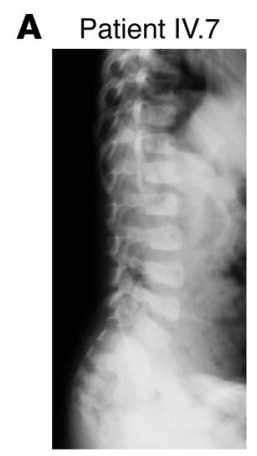

Patient IV.8

B

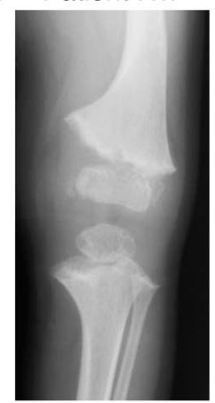

Patient IV.8

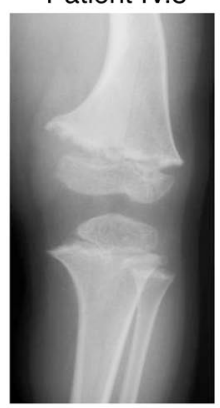

in childhood (Figure 1), with rhizomelic shortening especially of the lower limbs and genu varum deformities secondary to bowing of the femora, tibiae, or both (5). Importantly, the unusual pear-shaped vertebrae of growth in childhood are distinctive but not severe when compared with other SEMDs (1). The modeling defects improve spontaneously by early adolescence, yet affected individuals remain shorter than unaffected siblings. Additionally, bowing deformities predispose to osteoarthritis, especially of the knees (5). The molecular defect causing SEMD MO was initially investigated, in 1997, by genetic linkage studies using polymorphisms associated with 12 candidate genes, including COL2A1, COL9A1, COL9A2, COL9A3, COL10A1, COL11A1, COL11A2, PSACH, FGFR3, CRTL1, CMP, and PTHRP (11). Recombination between the disease and intragenic polymorphisms or significantly negative $\log _{10}$ of odds (LOD) scores $(<2.0)$ excluded these as the causative gene for SEMD $\mathrm{DO}_{\mathrm{MO}}$. To elucidate the etiology and pathogenesis of SEMD $\mathrm{D}_{\mathrm{MO}}$, we performed a genome-wide linkage

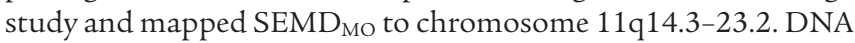
sequence analysis of a candidate gene encoding MMP13 identified a mutation in its proregion domain. This mutation leads to intracellular autoactivation and degradation of the mutant proenzyme, which is likely to be misfolded, with the resulting MMP13 deficiency causing SEMD MO.

\section{Results}

Chromosomal localization of SEMD $D_{M O}$ and identification of MMP13 mutation. To delineate the chromosomal location of SEMD $\mathrm{MO}_{\mathrm{MO}}$, we undertook a genome-wide search using leukocyte DNA from the 32 living individuals (Figure 2) of the kindred in whom SEMD had been inherited as an autosomal dominant disorder in 4 generations $(5,11)$. We used PCR together with fluorescently labeled primers to detect 382 microsatellite polymorphisms arranged in chromosome-specific sets, with an average intermarker distance of $10 \mathrm{cM}(12)$. This yielded a peak LOD score of 4.62 , at $0 \%$ recombination, between D11S898 and SEMD ${ }_{M O}$

\section{Figure 1}

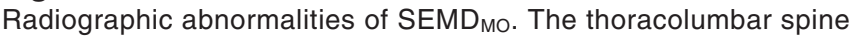
and left knee of patients IV.7 and IV.8 (Figure 2) at age 3.8 years are shown. (A) Pear-shaped vertebrae, with greatest vertical height anteriorly as well as bulbous anterior superior-inferior contours that are more pronounced in the lumbar region in patient IV.7. Disc spaces are increased posteriorly in both patients. These pearshaped vertebrae in early childhood evolve to mild platyspondyly with irregular superior and inferior margins in adults. (B) The left knees of patients IV.7 and IV.8 show femoral bowing, metaphyseal flaring and irregularity, and widened growth plates. The epiphyses are small and flat, and those of patient IV.7 have slightly irregular margins. There is rhizomelic shortening in patient IV.8. These findings of rhizomelic shortening, femoral bowing, and metaphyseal flaring and irregularity, with widened growth plates (physes), are consistent with those in SEMD.

(Table 1). In addition, an analysis of individual recombinants

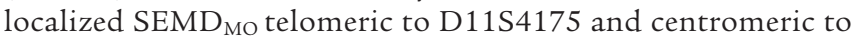
D11S908 (Figure 2). Hence, SEMD ${ }_{\mathrm{MO}}$ mapped to chromosome 11q22. To refine the critical region, 9 additional polymorphic microsatellite loci were investigated. This revealed peak LOD scores of greater than 3 between SEMD MO $_{\text {and }} 4$ loci (D11S1333, D11S1339, D11S927, and D11S4206; Table 1), confirming the SEMD $_{\mathrm{MO}}$ location at $11 \mathrm{q} 14.3-23.2$. An analysis of recombinants

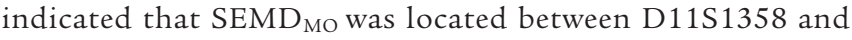
D11S4206 (data not shown), which span an interval of approximately $20 \mathrm{Mb}$ (Figure 3). The ENSEMBL database (http://www. ensembl.org) revealed this interval to contain approximately 160 genes (145 confirmed transcripts and 15 predicted transcripts)

\section{Table 1}

Two-point linkage analysis between $\mathrm{SEMD}_{\mathrm{MO}}$ and 17 loci from chromosome 11q14-q23
Locus

11cen $\uparrow$

D11S1314C
D11S937C
D11S901C
D11S4175C
D11S1332
D11S1358
D11S4118
D11S1333
D11S917C
D11S898C
D11S1339
D11S927
D11S4206
D11S1778
D11S1893
D11S4090C
D11S908C

11qter $\downarrow$

$\begin{array}{ll}0.96(0.15) & - \\ 1.91(0.10) & 6.9 \\ 2.96(0.05) & 4.4 \\ 2.96(0.05) & 6.4 \\ 2.95(0.05) & 0.0 \\ 2.69(0.05) & 0.0 \\ 2.61(0.00) & 1.2 \\ 4.62(0.00) & 0.7 \\ 2.96(0.00) & 2.7 \\ 4.62(0.00) & 2.2 \\ 4.32(0.00) & 1.7 \\ 4.02(0.00) & 2.2 \\ 3.04(0.05) & 0.0 \\ 1.84(0.10) & 1.7 \\ 2.32(0.05) & 1.1 \\ 1.62(0.15) & 0.5 \\ 1.27(0.15) & 3.8\end{array}$

\section{Peak LOD score $(\theta)^{A} \quad$ Distance between Ioci $(\mathrm{cM})^{\mathrm{B}}$}

ALOD scores were calculated under an autosomal dominant mode of inheritance, a penetrance of $100 \%$, and a phenocopy rate of $0 . \theta$ represents the recombination fraction. BDistances and the order of loci (11cen to 11qter) are from reported maps databases (ref. 50 and http://ensembl.org/Homo sapiens/mapview?chr=11). CPolymorphic loci $(n=8)$ used in the first stage; the remaining 9 loci were utilized to further delineate the critical interval. 


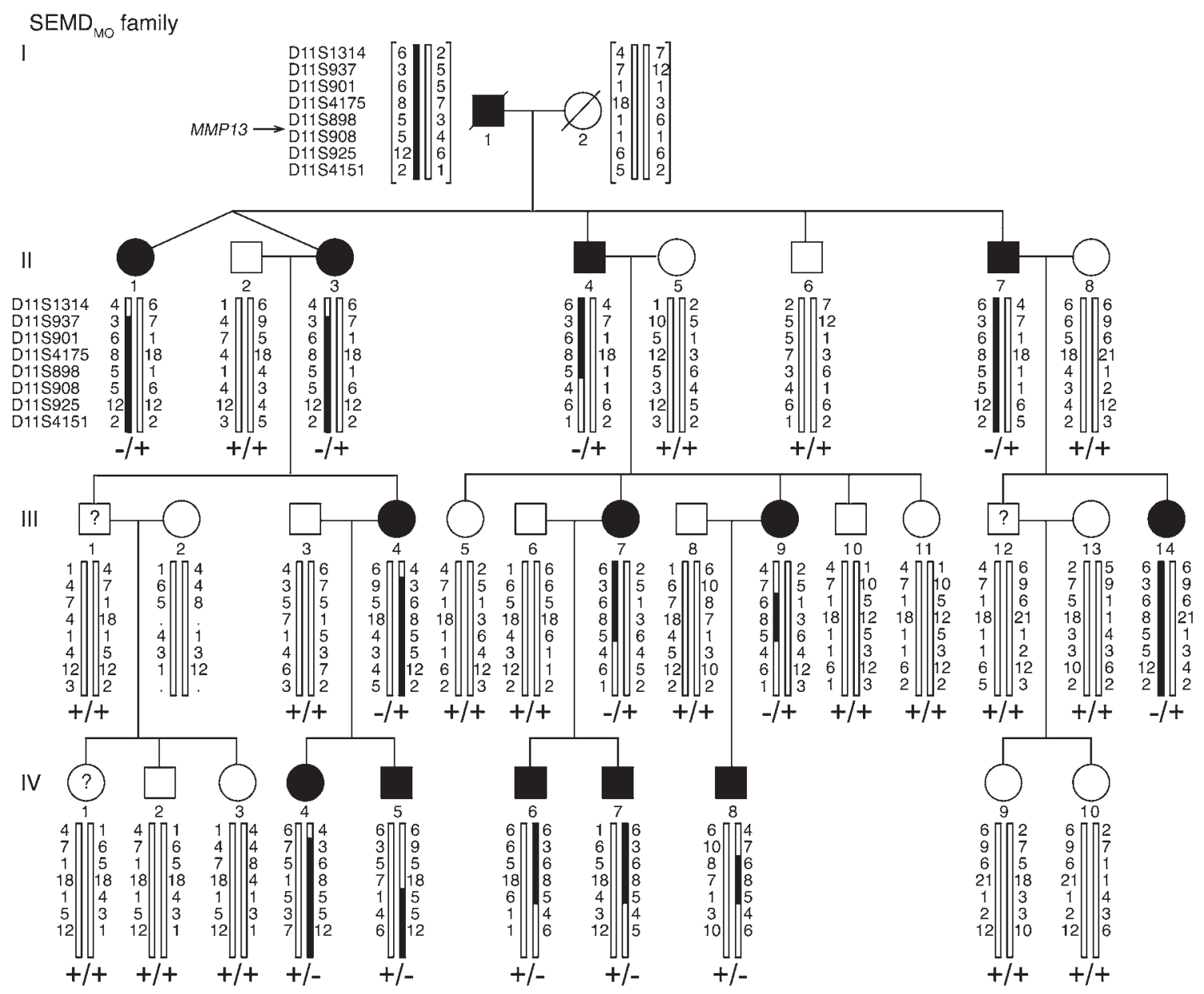

Figure 2

Pedigree of family segregating for SEMD ${ }_{\mathrm{MO}}$ and chromosome $11 \mathrm{q}$ loci. SEMD $\mathrm{MO}$ is segregating in the affected individuals II.7 and III.14 with the haplotype [6,3,6,8,5,5,12,2], defined by the loci: 11cen - D11S314 - D11S937 - D11S901 - D11S4175 - D11S898 - D11S908 - D11S925 - D11S4151 - 11qter. However, recombinants between SEMD $\mathrm{MO}_{\mathrm{M}}$ and the centromeric loci D11S314, D11S937, D11S901, and

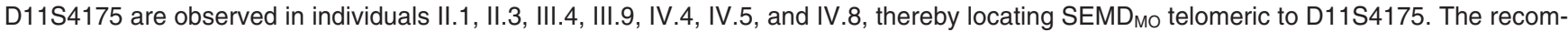
binants observed between SEMD $\mathrm{MO}_{\mathrm{M}}$ and the telomeric loci D11S4151, D11S925, and D11S908 in the affected individuals II.4, III.7, III.9, IV.6,

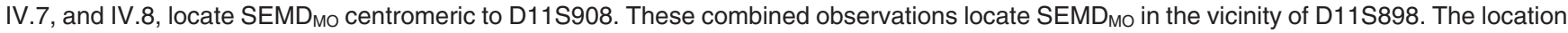
of MMP13, $1.69 \mathrm{Mb}$ telomeric to D11S898 (Figure 3), is indicated by the arrow. The presence, in each individual, of the wild-type F56 or the mutant S56 is represented by + and -, respectively. The pedigree has been revised from the original description $(5,11)$ to indicate those family members who yielded information for the localization of SEMD $\mathrm{MO}_{\mathrm{MO}}$. Squares, males; circles, females; open symbols, unaffected individuals; filled symbols, affected individuals; (?), uncertain SEMD ${ }_{\text {Mо }}$ phenotype; black bars, affected haplotypes; white bars, unaffected haplotypes. The paternal haplotypes are on the left, and the maternal haplotypes are on the right. Deduced haplotypes are within brackets.

that included a cluster of 9 genes encoding for MMPs. MMPs belong to a family of zinc metalloendopeptidases, comprising more than 20 members, that degrade components of the ECM $(13,14)$. The MMPs clustered on chromosome 11q22.3 include $M M P 7$ (matrilysin), MMP20 (enamelysin), MMP8, MMP1, MMP12 (macrophage elastase), MMP13 (collagenase 3), MMP10 (stromelysin 2), MMP3 (stromelysin 1), and MMP27 (unknown function) (Figure 3$)$. We considered MMP13 $(15,16)$ the best candidate for $\mathrm{SEMD}_{\mathrm{MO}}$, as it preferentially degrades collagen type II (17), abnormalities of which result in Strudwick type SEMD $(1-3,7)$. In addition, MMP13 is specifically expressed in the hypertrophic chondrocytes and osteoblasts of fetal bones, in remodeling bone postnatally, and in joints affected with osteoarthritis and rheumatoid arthritis $(17,18)$. These combined features supported our hypothesis that an MMP13 abnormality caused SEMD ${ }_{\mathrm{MO}}$, and we undertook DNA sequence analysis of the MMP13 gene (Figure 4), which consists of 10 exons that span $12.5 \mathrm{~kb}$ of genomic DNA (19). Our analysis revealed a missense mutation, Phe56Ser (F56S) (Figure 5), that involved the proregion domain of MMP13 (Figure 4). The mutation was confirmed by $M s p$ I restriction endonuclease analysis and demonstrated to cosegregate with $\mathrm{SEMD}_{\mathrm{MO}}$ (Figures 2 and 5) and to be absent in 110 alleles of 55 unrelated normal individuals, thereby indicating that it was not a functionally neutral polymorphism. Furthermore, the F56 residue is evolutionarily conserved in other MMP13s (Figure 3) and in human MMP1, MMP2, MMP7, MMP8, MMP20, and MMP27, thereby further indicating that the F56S abnormality is likely to be functionally significant. 


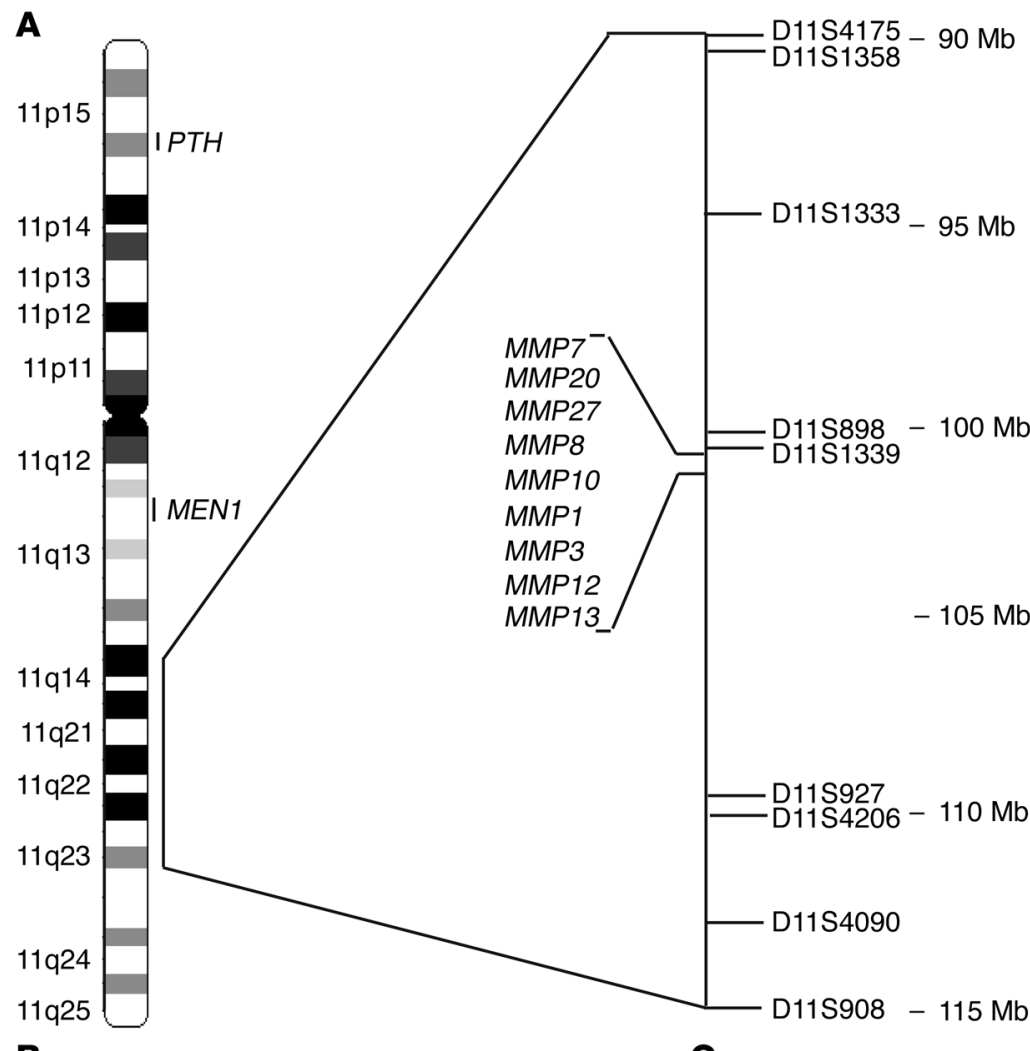

B

Homo sapiens

Mus musculus

Rattus norvegicus

Equus caballus

Bos taurus

Xenopus laevis

Danio rerio

$\mathrm{SEMD}_{\mathrm{MO}}$ kindred

\begin{tabular}{|c|c|c|}
\hline * & & * \\
\hline LREMQSFFGLEVTGK & $M M P 1$ & LKQMQEFFGLKVTGK \\
\hline LREMQSFFGLEVTGK & $M M P 7$ & LKEMQKFFGLPITGM \\
\hline LREMQSFFGLDVTGK & MMP 8 & LKEMQRFFGLNVTGK \\
\hline LREMQSFFGLEVTGK & $M M P 13$ & LREMQSFFGLEVTGK \\
\hline LREMQSFFGLEVTGK & $M M P 20$ & IKELQAFFGLQVTGK \\
\hline LREMQSFFGLEVTGR & $M M P 27$ & IREMQAFFGLTVTGK \\
\hline
\end{tabular}
LKEMQSFFGLEVTGK

LREMQSFSGLEVTGK

\section{Figure 3}

Schematic representation of chromosome 11 with Giemsa bands, showing the positions of 8 polymorphic loci from 11q14.3-23.2 that helped define the location of SEMD $\mathrm{MO}_{\text {MO }}$ (A) The locus order was derived from consensus maps (http://www. ensembl.org/Homo_sapiens/mapview? chr=11; http://www.broad.mit.edu/tools/data/data-human. $\mathrm{html}$ ). Recombinants between $\mathrm{SEMD}_{\mathrm{MO}}$ and the centromeric loci D11S4175 and D11S1358 and the telomeric loci D11S4206 and D11S908 (Figure 2 and Table 1) located SEMD ${ }_{M O}$ to an approximate 17-cM (equivalent to a 20-Mbp) interval on chromosome 11q14.3-23.2. Within this interval, SEMD $\mathrm{MO}_{\mathrm{M}}$ cosegregated with 6 loci (Table 1), and 4 of these (D11S1333, D11S898, D11S1339, and D11S927) revealed peak LOD scores greater than +3 at $0 \%$ recombination. This $20-\mathrm{Mbp}$ interval contains 160 genes (http://www.ensembl.org/Homo_sapiens/ mapview?chr=11) that include a cluster of 9 genes encoding MMPs, located at 11q22.3. These are MMP1, -3, -7, -8, -10, -12, -13, -20, and -27. The MMP13 gene, which is also referred to as collagenase 3, harbored a missense mutation, F56S

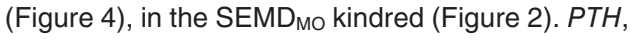
parathyroid hormone; MEN1, multiple endocrine neoplasia type 1. (B) The human F56 residue (asterisk) is evolutionarily conserved in the MMP13 of mammals, amphibia, and teleost fish (http:// www.broad.mit.edu/tools/data/data-human.html). (C) The MMP13 F56 residue (asterisk) is also conserved in other members of the MMP family (e.g., MMP-1, -3, -7, -8, -10, -12, -13, -20, and -27) in humans and similarly in mouse, rat, horse, rabbit, and cow (data not shown) (http://www.ncbi.nlm. nih.gov/gquery/gquery.fcgi?term $=\mathrm{mmp} 13$ ). Thus, the F56S mutation in the SEMD ${ }_{\text {MO }}$ kindred likely represents a significant change.
Analysis of expressed wild-type and mutant F56S MMP13. Substitution of the hydrophobic F56 with the hydrophilic S56 in the mutated

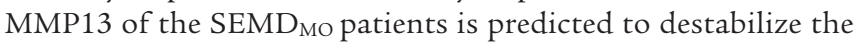
folded structure of the protein intracellularly $(20,21)$. Misfolded F56S proMMP13 could then lead to aberrations in intracellular transport and autoactivation and retention and/or degradation in the ER (22). We investigated some of these possibilities by expressing, in human embryonic kidney 293 (HEK293) cells, wild-type or mutant F56S MMP13 cDNAs, which had been subcloned into pcDNA3.1 with or without a C-terminal MycHis tag (Figure 6). The expected molecular mass of expressed proMMP13 protein (including the MycHis tag of approximately $5 \mathrm{kDa}$ ) is approximately $60 \mathrm{kDa}$; removal of the prodomain would result in an active form with molecular mass of approximately $45 \mathrm{kDa}(20)$. In vitro translation (IVT) (23) of wild-type and F56S proMMP13 cDNAs showed similar protein products (Figure 6A). Conditioned medium from HEK293 cells transiently transfected with wild-type or F56S were used for Western blot analysis, which revealed the secretion of full-length untagged or MycHis-tagged wild-type MMP13 but only trace amounts (or none) of full-length untagged or MycHis-tagged F56S proMMP13 (Figure 6, B-D). Instead, degraded misfolded protein fragments of approximately $25-30 \mathrm{kDa}$ of the mutant protein were secreted. These results were found to be the same using 3 different anti-MMP13 antibodies (Figure 6). The F56S is thus abnormally secreted as well as activated and degraded intracellularly.

We investigated further mechanisms that might account for these findings. Among the most likely possibilities are: a misfolding of F56S MMP13 in the ER such that the orderly progression to the Golgi apparatus and secretion does not take place, but instead the protein is dislocated to the cytosol and degraded in proteasomes $(24,25)$; or a delayed secretion of the misfolded protein that is susceptible to intra- or extracellular autoactivation (26) and autodegradation. To resolve these issues, we first cultured transfected HEK293 cells in the presence of the proteasome inhibitor lactacystin $(10 \mu \mathrm{M})(27,28)$. No alteration of the pattern of intracellular F56S MMP13 was observed by Western blotting (data not shown), thereby suggesting that proteasomes were not involved in processing of the mutant protein. We next used monensin, a disruptor of the Golgi apparatus (29), to block secretion. We found that monensin abolished the secretion of fulllength wild-type proMMP13 as well as the low-molecular-mass degradation fragments of F56S MMP13 (Figure 7A). In cell lysates from F56S MMP13 transfectants in the presence of monensin, 


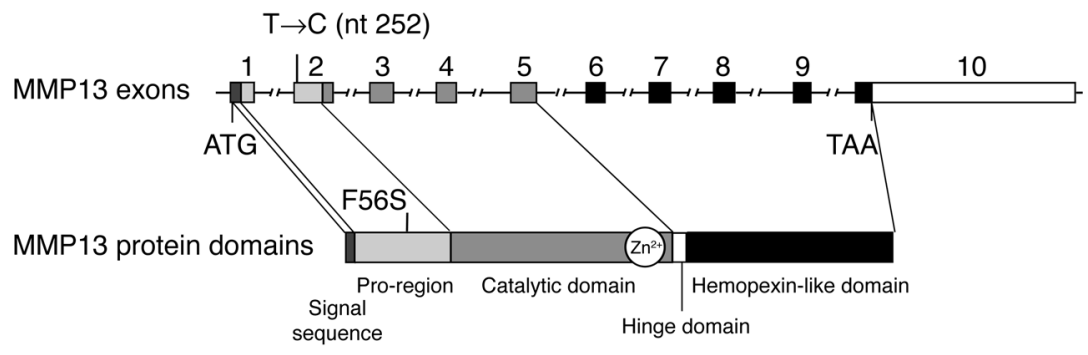

Figure 4

Schematic representation of the genomic structure of the MMP13 gene illustrating locations of the SEMD ${ }_{\text {MO }}$ mutation and protein domains. The 1,413-bp MMP13 coding region yields a 471-amino acid preproenzyme. The signal sequence consists of 19 amino acids that are removed on processing and are not present in the secreted proenzyme $(15,16)$. The pro- and catalytic domains comprise 84 and 166 amino acids, respectively. The catalytic domain has a zinc-binding motif (HEXXHXXGXXH) that contains 3 histidine residues that bind the catalytic zinc ion at the active site $(15,16,19,36)$. The proregion contains a cysteine residue (Cys77) that ligates this catalytic zinc ion to maintain the latency of proMMP13. An 11-amino acid proline-rich linker peptide (hinge domain) connects the catalytic domain and hemopexin domain, which consists of 191 amino acids. The position of the $\mathrm{T} \rightarrow \mathrm{C}$ transition at nucleotide 252 of exon 2, detected in the SEMD $\mathrm{MO}_{\text {kindred (Figure 2) }}$ and found to result in the F56S mutation (Figure 5) in the proregion, is shown. The shading or hatching of each protein domain indicates its exonic origins.

low levels of full-length proMMP13 protein were seen, as well as more abundant low-molecular-mass fragments. In contrast, in cell lysates from cultured wild-type MMP13 transfectants, only low levels of proMMP13 were found. These results are consistent with the idea that F56S MMP13 is autoactivated and degraded within the ER/Golgi and not in proteasomes, as is the case for other misfolded mutant human proteins, such as $\alpha 1$-antitrypsin $\mathrm{Z}$ (28). Moreover, these experiments indicate that F56S MMP13 is degraded intracellularly rather than extracellularly.

In order to determine whether F56S MMP13 is degraded primarily because of delayed secretion and then partial cleavage by some other intracellular proteinase, we constructed catalytically inactive MMP13 mutants. The glutamic acid residue $(\underline{E})$ in the sequence (HEXXGH) of the zinc-binding domain (Figure 4) is critical for catalysis by metalloproteinases, and $\mathrm{E} \rightarrow \mathrm{A}$ mutants of other MMPs are catalytically inactive $(30,31)$. We therefore constructed 2 new MMP13 mutants, E204A and the compound F56S/E204A, in pcDNA3.1 and transfected them into HEK293 cells. Both the E204A MMP13 and the compound F56S/E204A MMP13 were expressed at levels similar to those of wild-type E204 MMP13 and were normally secreted (Figure 7B). Thus, mutation of the catalytically active E204 residue to A204 abolished the intracellular autoactivation and autodegradation that resulted from the F56S mutation. The effects of $p$-aminophenyl mercuric acetate (APMA), which has been shown to activate several MMPs $(13,32)$, were also assessed. Incubation with APMA resulted in activation of wild-type MMP13 with subsequent autodegradation to fragments of lower molecular mass; APMA did not affect the molecular mass of E204A MMP13 or that of the compound mutant F56S/E204A MMP13 (Figure 7, C and D). These studies, which utilized the epithelial renal cell line HEK293, were confirmed using the transformed human chondrocyte line T/C-28a4 (33) (data not shown). These results dem-

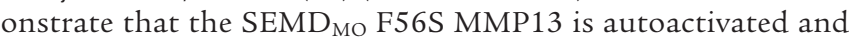
autodegraded intracellularly. Indeed, the catalytically inactive E204A mutant can be envisaged as "rescuing" the abnormal intracellular degradation of the SEMD $\mathrm{MO}^{-}$ associated F56S mutant MMP13. Moreover, abnormal secretion and degradation of F56S MMP13 are likely due to misfolding in the cell that interferes with maintenance of the inactive proMMP13, rather than the misfolding interfering with the secretion itself. This is further supported by our experiments with mutant C77S MMP13 (Figure 7E). Studies of MMPs other than MMP13 $(32,34)$ have shown that within the prodomain, a cysteine residue downstream from the conserved residues orthologous to F55 and F56 in MMP13 folds over to bind to the catalytic zinc (Figure 4) to form a "cysteine switch," essential for keeping proMMPs inactive prior to secretion (35). Indeed, the $\mathrm{C} 77$ residue of MMP13 is included in the sequence PRCGV, which is designated as the "cysteine-switch box," a feature common to MMPs and ADAM (a disintegrin and metalloproteinase) proteinases $(34,35)$. We therefore constructed the C77S mutant MMP13 for transfection into HEK293 cells. The resultant autoactivation and secretion of autocatalytic fragments was observed in a pattern indistinguishable from that

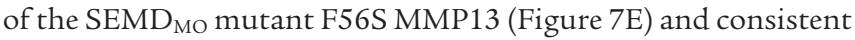
with a cysteine-switch box role for this motif in MMP13.

Coexpression of wild-type and mutant F56S MMP13, using stable and transient transfectants. SEMD $\mathrm{DO}_{\mathrm{MO}}$ patients are heterozygotes and have both wild-type and mutant F56S copies of MMP13 (Figures 2 and 5). To investigate the dominant nature of the mutant F56S MMP13, we first established stable transfectants of wild-type and mutant F56S MMP13 constructs in HEK293 cells. The stable and transient transfectants behaved similarly (Figure 7F). Full-length wild-type MMP13 was present in conditioned medium, but only low-molecular-mass fragments of F56S MMP13 were detected. In addition, proMMP13 in the medium from the stable transfectants of wild-type MMP13 was activatable by APMA (data not shown) in a manner similar to that observed from the transient transfectants (Figure 7, C and D). To explain the dominant effects of the S56 mutation, we postulated that the activated mutant MMP13 allele product before it was completely degraded could intracellularly activate the wild-type allele proMMP13 product and lead to degradation and decreased secretion of potentially activatable proMMP13. To investigate this, we transiently transfected a C-terminal Flagtagged wild-type proMMP13 construct that could be activated by APMA into HEK293 cells, which had been stably transfected with empty-vector, wild-type, or F56S MMP13 cDNA (Figure 7G). Fulllength wild-type MMP13, identified with the anti-Flag antibody, was present in cell lysates and conditioned medium from empty vector or wild-type MMP13 clones. In contrast, low-molecular-mass fragments of wild-type MMP13 were detected with anti-Flag antibody only in conditioned medium and cell lysates from the stable clones that expressed F56S MMP13. The lower-molecular-mass fragments secreted from cells stably transfected with the F56S MMP13 construct were retained intracellularly in the presence of monensin. These results indicate that the wild-type Flag-tagged MMP13 is activated by autoactivated F56S MMP13 within the cell, presumably in the ER/Golgi. In heterozygotes, this would result in decreased levels of secreted proMMP13 that could be active 
A

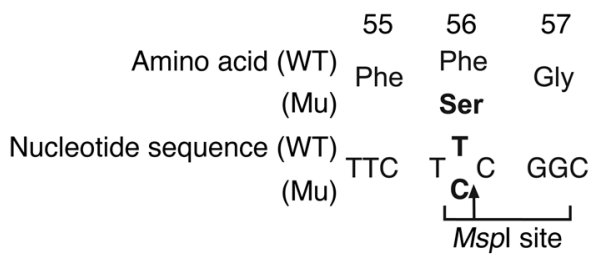

B
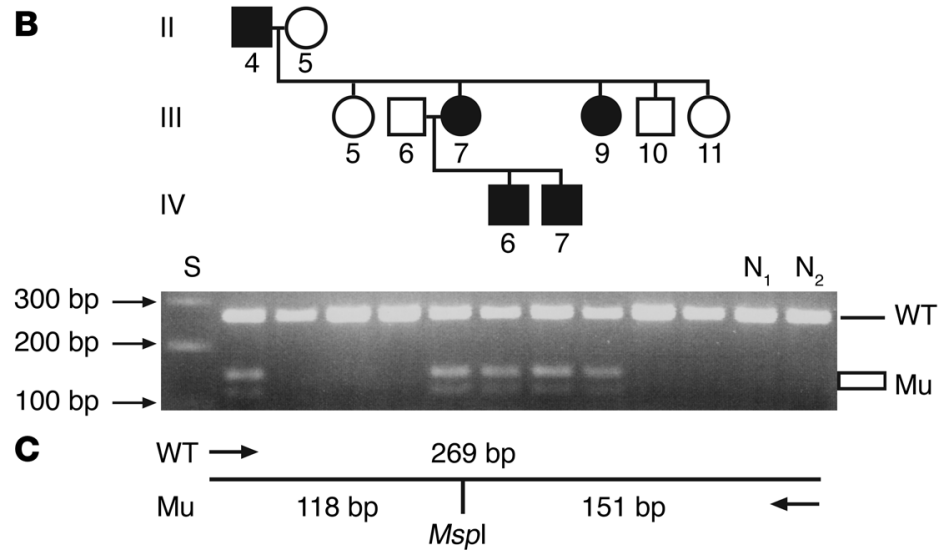

Figure 5

Detection of mutation in exon 2 by restriction enzyme analysis. (A) DNA sequence analysis of the affected female III.7 revealed a $\mathrm{T} \rightarrow \mathrm{C}$ transition at codon 56. The wild-type sequence at codon 56 is TTC encoding a phenylalanine (Phe, F) residue, whereas the mutant $(\mathrm{Mu})$ is TCC encoding for a serine (Ser, S) residue. This missense mutation resulted in the gain of an Mspl restriction site (C/CGGC), which facilitated its detection in the family with SEMD $\mathrm{MO}_{\mathrm{MO}}$ (B). (C) The restriction enzyme map of the PCR products shows that the wild-type DNA sequence results in a 269-bp product, whereas the mutant, which has one Mspl site, is associated with cleaved PCR products of $118 \mathrm{bp}$ and $151 \mathrm{bp}$. Analysis of the SEMD $\mathrm{MO}_{\mathrm{O}}$ family (Figure 2) revealed that the affected members (II.4, III.7, III.9, IV.6, and IV.7; shown [filled symbols]) were heterozygous for the F56S mutation and that the unaffected members (II.5, III.5, III.6, III.10, and III.11; shown [open symbols]) were homozygous for the wild-type sequence. Standard size markers (S), in the form of a 100-bp ladder, are shown. Cosegregation of this mutation with the disease in the family (Figure 2), in conjunction with its absence in 110 alleles from 55 normal individuals ( $\mathrm{N}_{1}$ and $\mathrm{N}_{2}$ are shown) indicates that it is not a common DNA sequence polymorphism.

in extracellular proteolysis. We therefore suggest that in vivo, in individuals with SEMD $\mathrm{MO}_{\mathrm{MO}}$, within the cell the activated product of the mutant proMMP13 allele, before it is extensively degraded, activates the product of the wild-type allele. This would then result in lower levels of secreted wild-type MMP13 than predicted on the basis of haploinsufficiency alone and therefore account for the dominant nature of the SEMD $\mathrm{MO}_{\mathrm{MO}}$ mutation.

These considerations assume, based on the proteolytic assays described, that the proMMP13 activated within the cell is degraded to fragments that no longer have collagenolytic or nonspecific proteinase (gelatinase) activity. Indeed, this was confirmed by measuring proteolytic activity of secreted proteins (Figure 8). Conditioned medium from stable transfectants was concentrated, and proteolytic activity was measured in collagenase and gelatinase assays using native type I collagen or denatured type I collagen (gelatin) as substrates. There was abundant collagenase and gelatinase activity in the conditioned medium from wild-type MMP13 stable transfectants, but neither collagenase nor gelatinase activity was detected in the conditioned medium from F56S
MMP13 stable transfectants (Figure 8). Similar results were obtained using type II collagen as substrate (data not shown). These results are consistent with the idea that the decreased extracellular proteolytic activity is the consequence of the F56S mutation.

Structural analysis of MMP13 pro- and catalytic domains. To understand the mechanisms for the effects of the abnormal folding of proMMP13 on enzyme function, it would be necessary to obtain and compare the crystal structure of wildtype and mutant proMMP13. Although there are published data from studies using X-ray crystallography and NMR concerning the catalytic, hinge, and C-terminal domains of MMP13, the structure of the prodomain of MMP13 has not been reported (36). We therefore modeled the prodomain and catalytic domains of wild-type and mutant MMP13 using the available structures of proMMP2 (progelatinase A) and proMMP3 (prostromelysin-1), together with the sequences and other available structural features of MMP13 as templates $(30,36)$. Our analysis revealed that in wild-type MMP13, the F56 side chain lies buried in the interface between the prodomain and the catalytic domain (Figure 9A). At that location, F56 maintains aromatic contacts with F55 and F222, hydrophobic contacts with M52 and P223, and van der Waals interactions with D81. The loss of the bulky side chain of F56 in the mutant MMP13 would then generate a hydrophobic cavity $(20,21)$ that cannot be filled by the smaller, polar side chain of S56, thereby resulting in a more open and unstable structure (Figure 9B). Based on these considerations, we constructed F55T and F222S MMP13 mutant cDNAs to express in HEK293 cells. The F55T mutant behaved identically to F56S (Figure 7H) as predicted from the modeling (Figure 9), whereas the F222S mutation appeared to have weaker although definite effects on MMP13 stability (Figure 7H, arrow). Further validation of the model was obtained by constructing and expressing other proMMP13 mutants involving residues M52, D81, and P223 (Figure 9) (data not shown).

\section{Discussion}

Our studies have revealed that a missense mutation, F56S, of MMP13 causes SEMD ${ }_{\text {MO }}$ (Figure 5). This mutation resulted in intracellular autodegradation such that only proteolytically inactive, low-molecular-mass fragments of F56S MMP13 were secreted (Figures 6 and 7). These secreted fragments of low molecular mass were demonstrated, using native or denatured collagens as substrates (Figure 8), to have no proteolytic activity. Moreover, our use of catalytically inactive mutants E204A and C77S (Figure 7) indicated that the abnormal secretion and degradation of the F56S MMP13 was likely to be due to intracellular misfolding of the mutant protein together with autoactivation. The glutamic acid residue (E) in the zinc-binding domain (Figure 4) of metallopeptidases participates in the mechanism of catalysis by promoting the attack of a water molecule on the carbonyl carbon of the substrate $(31,32,36,37)$. Substitution of an alanine (A) for this glutamic acid (E) in the wild-type or mutant MMP13 renders the protein catalytically inactive. The abnormal intracellular degradation and altered secretion of F56S MMP13 is thus "rescued" by the E204A mutation (Figure 7). The role of the C77 residue is equally important. During synthesis of wild-type MMP13, proper folding of the 
A

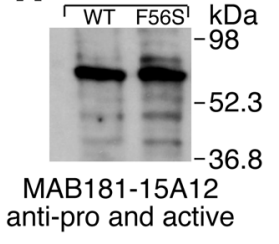

C

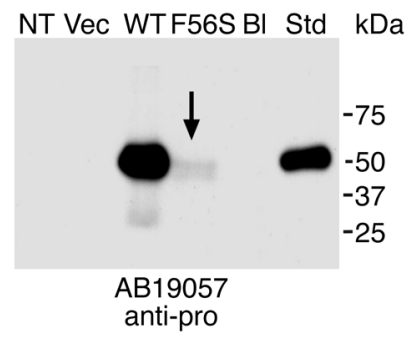

B

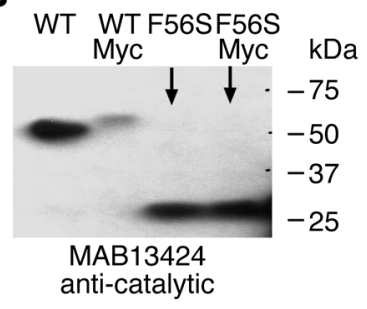

D

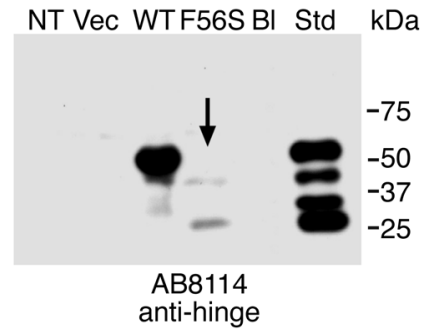

prodomain permits the $\mathrm{C} 77$ to bind to the catalytic zinc and prevent activation prior to secretion. In the mutant F56S MMP13, binding of $\mathrm{C} 77$ to the catalytic zinc does not take place, and degradation occurs within the ER/Golgi (Figure 7). The manner in which misfolding of F56S MMP13 may occur was further investigated by modeling the structure of the pro- and catalytic domains of MMP13 (Figure 9). This revealed that the F56S mutation responsible for $\mathrm{SEMD}_{\mathrm{MO}}$ is an interesting example of a "cavity-forming" mutation, whose relevance to the folding and stability of globular proteins has been previously described $(20,21)$. However, it is important to note that introduction of different substitutions for specific hydrophobic residues can have different effects on structural stability. For example, the structural stability of phage T4 lysozyme is dependent on its hydrophobic core, and introduction of substitutions of Ala for different hydrophobic residues decreases the protein stability to different extents $(37,38)$. Thus, although we have identified several important residues in the hydrophobic core of MMP13 that affect protein stability, it is possible that motifs in the MMP13 prodomain other than those in the hydrophobic core could also function in maintaining the latency of proMMP13. In order to resolve these issues, it will be necessary to obtain the crystal structure of the catalytically inactive E204A MMP13 as well as the compound mutant F56S/E204A MMP13.

The results of our study help to elucidate the role of the MMP13 missense mutation F56S in causing SEMD $\mathrm{MO}_{\mathrm{M}}$. The clinical and radiographic features of $\mathrm{SEMD}_{\mathrm{MO}}$ (Figure 1) and its childhood onset are consistent with abnormalities of human skeletal development and remodeling. Among the MMPs, MMP13 has been shown to have important roles in endochondral and intramembranous ossification during human fetal bone development. MMP13, expressed in hypertrophic chondrocytes and osteoblasts during embryonic ossification $(17,18,39-41)$, can degrade collagen types I, II, III (17), and X (42), as well as noncollagenous proteins such as aggrecan (43). During endochondral ossification, chondrocytes proliferate and elaborate an ECM composed principally of collagen type II. As chondrocytes differentiate, they synthesize collagen $\mathrm{X}$, and the cartilage matrix is calcified and eventually degraded. The loss of collagen at the hypertrophic zone in the growth plate is associated with increased MMP13

\section{Figure 6}

Analysis of expression of wild-type and F56S MMP13 cDNAs in transfected HEK293 cells. (A) IVT of MMP13 cDNAs. A product of similar molecular mass, approximately $65 \mathrm{kDa}$, was produced with either wild-type or mutant F56S MMP13 cDNA as template. (B) Western blot analysis of secretion of expressed wild-type and mutant F56S cDNAs in HEK293 cells. Full-length untagged and or Myc-tagged wild-type MMP13 were secreted into conditioned medium, whereas only fragments of F56S MMP13 of low molecular mass were secreted. (C) As in $\mathbf{B}$, but anti-prodomain antibody was used. (D) As in $\mathbf{B}$, but anti-hinge domain antibody was used. In $\mathbf{C}$ and $\mathbf{D}$, autodegraded recombinant MMP13 was used as standard. NT, no transfection; Myc, MycHis-tag; $\mathrm{Vec}$, vector only; BI, blank lane; Std, human MMP13 standard, autoactivated and partially degraded.

activity. This is followed by partial resorption of the calcified cartilage, leaving cartilaginous trabeculae on which osteoblasts elaborate woven bone that is subsequently resorbed and replaced by mature trabecular bone (18). Thus, MMP13 deficiency would prevent orderly ECM degradation in developing growth plates with accumulation of collagens type II and X, and disturbed endochondral ossification. Indeed, studies of Mmp13-null mice have revealed defects in growth plate cartilage with increased hypertrophic domains and delayed endochondral ossification during embryonic development $(40,41)$. The growth plate phenotype of the Mmp13-null mice persists into adult life and is consistent with that seen in patients with $\operatorname{SEMD}_{\mathrm{MO}}(5)$. However, in humans, the SEMD ${ }_{\text {MO }}$ mutation is dominant (Figures 2 and 5), and this contrasts with the situation in heterozygous $\left(\mathrm{Mmp}^{13^{+-}}\right)$ mice, which have normal growth plates $(40,41)$. Thus, haploinsufficiency of MMP13 alone cannot account for the phenotype of SEMD ${ }_{\mathrm{MO}}$, and based on our studies that mimicked heterozygosity by coexpression of wild-type and mutant MMP13 (Figures 7 and 8), we propose that the F56S enzyme, before it is fully proteolytically degraded, activates and degrades, intracellularly, the MMP13 product of the wild-type allele and thereby exerts a dominant action. Thus, our results, which have elucidated the

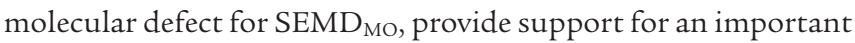
role for MMP13 in skeletal development.

These findings indicate that $\mathrm{SEMD}_{\mathrm{MO}}$, with regard to the rapidly emerging molecular-pathogenic classification of genetic disorders of the skeleton (2), belongs within group 3 , which consists of defects in folding and degradation of macromolecules. Group 3 includes the Torg type osteolysis syndrome, caused by MMP2 mutations (44), and X-linked spondyloepiphyseal dysplasia tarda, caused by sedlin mutations (45). Thus, SEMD ${ }_{M O}$ is the first heritable disorder associated with an MMP13 mutation, and it is likely that other MMP13 mutations could cause other allelic skeletal dysplasias featuring an SEMD phenotype and therefore analogous to type II collagenopathies $(1,2)$. Furthermore, the association of SEMD ${ }_{M O}$ with a missense MMP13 mutation, and that of Torg syndrome with deactivating MMP2 mutations, shows that rare skeletal dysplasias can involve MMPs and that a search for such abnormalities would be worthwhile.

\section{Methods}

Genotyping and linkage analysis. Venous blood was obtained from the 32 living individuals (13 affected and 11 unaffected members and 8 unaffected spouses) of the SEMD $\mathrm{MO}_{\mathrm{O}}$ kindred (Figure 1) following receipt of informed consent, using protocols approved by the research ethical 


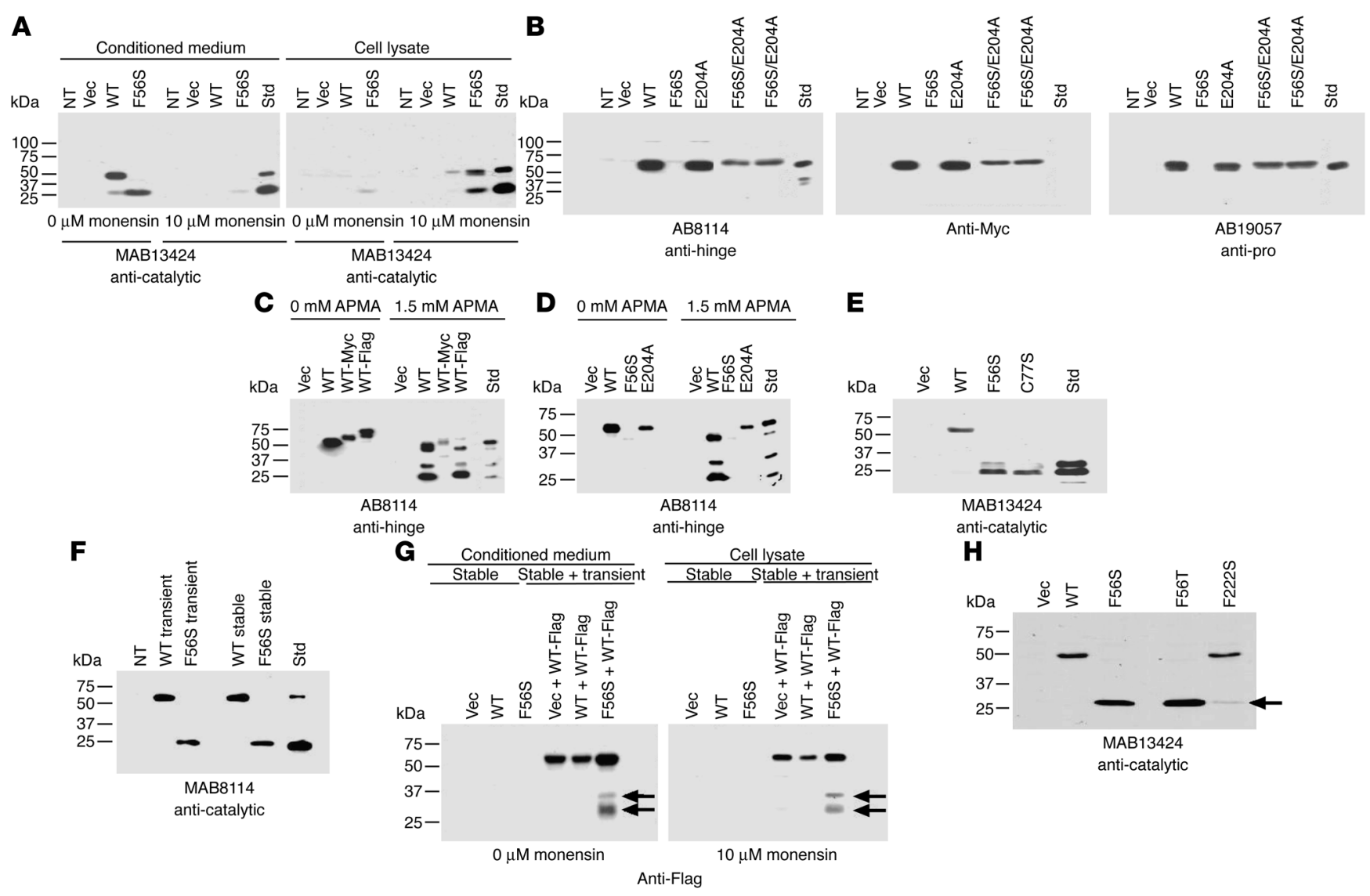

\section{Figure 7}

Analysis of expression of wild-type and mutant MMP13 cDNAs. All constructs have Myc tags. (A) Effects of monensin on secretion of WT and F56S MMP13. (B) Effects of E204A mutation. Two separate transfectants of F56S/E204A MMP13 are shown. (C) Activation of WT MMP13, with APMA, results in autodegradation and generation of lower-molecular-mass fragments. (D) Activation of WT MMP13 and E204A MMP13 are compared as in C. E204A MMP13 is not activated by APMA. (E) Secretion and autodegradation of mutant C77S MMP13. MMP13 mutants F56S and C77S are degraded to low-molecular-mass fragments. (F) Comparison of stable and transient transfectants. Protein products encoded by transient (HEK293T) and stable (HEK293) transfectants of WT and mutant F56S MMP13 cDNAs behaved similarly. (G) Transient cotransfection of Flag-tagged WT MMP13 cDNA into HEK293 cells stably expressing untagged WT or mutant F56S MMP13. Flag-tagged WT proMMP13 is normally secreted when expressed in cells stably transfected with empty vector or untagged WT MMP13 but is partially autoactivated and degraded (arrows) when expressed in cells stably expressing F56S MMP13. (H) Secretion and autodegradation of mutant F56S, F55T, and F222S MMP13. Transient transfection of F56S and F55T MMP13 cDNAs showed abundant low-molecular-mass fragments but no proenzyme. Transfection with F222S MMP13 cDNA revealed full-length proenzyme and low-abundance low-molecular-mass fragments (arrow). Results in all panels are based on experiments using conditioned medium unless otherwise stated.

committees of the Royal Postgraduate Medical School (London, United Kingdom); the Oxford Radcliffe Hospitals and University of Oxford; and Washington University School of Medicine. Leukocyte DNA was prepared using standard methods (12), and a genome-wide linkage analysis was performed using the Applied Biosystems Linkage Mapping Set (version 2.5) of 400 fluorescently labeled PCR primers to amplify polymorphic microsatellite loci spaced at an average intermarker distance of $10 \mathrm{cM}$, from the Généthon (http://www.genethon.fr) human linkage map (12). PCR was performed as previously described (46), and alleles were detected on a $5 \%$ denaturing polyacrylamide gel using an ABI PRISM 377XL DNA sequencer (Applied Biosystems) (12, 23). Alleles were sized by GeneScan Analysis Software (version 2.1) and scored by Genotyper software (version 2.0) (Applied Biosystems). Two-point LOD scores were calculated using the MLINK program of the FASTLINK (version 4.0P) package $(12,46)$, accessed via the Genetic Linkage User Environment (GLUE) interface (MRC UK Human Genome Mapping Project Resource Centre).
Allele frequencies were assumed to be equal, and the gene frequency and penetrance of SEMD ${ }_{M O}$ were taken as $10^{-4}$ and $100 \%$, respectively; varying these values had no significant effect on the linkage analysis. The locus order was derived from consensus maps (http://www.ensembl.org/ Homo_sapiens/mapview?chr=11 and http://www.broad.mit.edu/tools/ data/data-human.html).

$D N A$ sequence analysis of MMP13. Ten pairs of MMP13-specific primers were designed based on the genomic sequence (NCBI Entrez Nucleotide database; http://www.ncbi.nlm.nih.gov/entrez/query. $f(g i ? d b=$ Nucleotide) and used for PCR amplification of the 10 exons and 18 intron-exon boundaries, as previously described (23). One pair of genomic MMP13 primers (F [forward] and R [reverse]) were designed and used for each of the 10 MMP13 exons as follows: 1F 5'-GGCATCACCATTCAAGATGC-3'; 1R 5'-GGATTGGCAAGATACTCTACC-3'; 2F 5'-GGTGTCTTTTCTAGCGCTACC-3'; 2R 5'-GCCTATGATTTACCTGTAGG-3'; 3F 5'-AGAATTGTGAATTACACCCCTG-3'; 3R 5'-GTGCAT- 


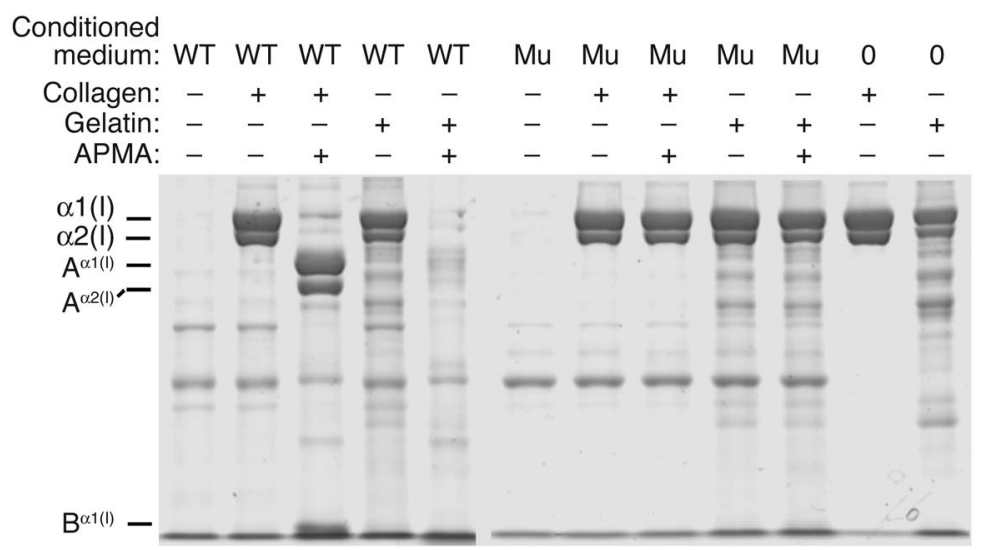

Figure 8

Assay for proteolytic activity of expressed, secreted wild-type and mutant F56S MMP13. Conditioned medium from HEK293 cells that had been stably transfected with either WT or mutant MMP13 (Figure 7, F and G) was used as source of enzyme. Type I collagen or gelatin was substrate, and following incubation, proteins were resolved by SDS-PAGE. Collagen $\beta$ and $\gamma$ components are not shown in this stained gel. Conditions for each lane are indicated. 0, no conditioned medium added; WT, wild-type MMP13; Mu, F56S MMP13; APMA, 1 mM APMA; Collagen, type I collagen; Gelatin, heated (denatured) type I collagen. WT MMP13 activated with APMA completely digested type I collagen to characteristic three-quarter fragments $\left(A^{\alpha 1(I)}\right.$ and $\left.A^{\alpha 2(I)}\right)$ and one-quarter fragments. The fragment $B^{\alpha 1(I)}$ is shown; at this concentration of acrylamide, the $B^{\alpha 2(l)}$ fragment migrated off the gel. Activated wild-type MMP13 also completely digested type I gelatin, but low-molecular-mass fragments of F56S MMP13 secreted into conditioned medium digested neither collagen nor gelatin.

CATTACCCTTAATTCC-3'; 4F 5'-CATACTAGTGTGTTCACTGTC-3'; 4R 5'-CCTGTGCTAAGTACTCCTGGATG-3'; 5F 5'-TACCTGGTCACTGGAATTCCC-3'; 5R 5'-ACCATAGAGAGACTGGATCCC-3'; 6F 5'-GCCTTTCACGACTACAGGTCC-3'; 6R 5'-GAATCTCAAGAGTACCTGTC-3'; 7F 5'-TGTTAGATTCTTCTGGCGCCTGC-3'; 7R 5'-CCTCTGTGGAAAGCTTACCTCTG-3'; 8F 5'-ATTTCACTCATTCCAGGTAG-3'; 8R 5'-ACAGATGGGTCAGTACCTAC-3'; 9F 5'-CCAGATGCTTTAAATGGACTG-3'; 9R 5'-GTTTTGAGGGGCTACAGAAGC-3'; 10F 5'-CTCTGAAATTGTCTTTATGC-3'; 10R 5'-ATTACCCCAAATGCTCTTCAGG-3'. DNA from an SEMD $_{\mathrm{MO}}$-affected individual (III.7; Figure 2) and from an unaffected unrelated subject was used, and the DNA sequences of the PCR products were determined as described (23), using a semiautomated system (ABI 373XL; Applied Biosystems). The DNA sequence abnormality was confirmed by MspI restriction enzyme analysis of genomic PCR products, obtained using MMP13 primers $2 \mathrm{~F}$ and $2 \mathrm{R}$ (sequences given above), and resolved by $2 \%$ agarose gel electrophoresis (23). The DNA sequence abnormality was also demonstrated to cosegregate with the disorder and to be absent as a common sequence polymorphism in DNA obtained from 55 unrelated normal individuals ( 27 males and 28 females).

Expression of MMP13 cDNAs. A full-length MMP13 cDNA was generated using PCR and the I.M.A.G.E. (http://image.llnl.gov) clone 796237 as template and subcloned in frame into the EcoRI site of pcDNA3.1 and also the pcDNA3.1 MycHis vector (Invitrogen Corp.) (23). Parent HEK293 or the variant HEK293T cells, which do not endogenously express MMP13, were transfected using Lipofectamine Plus (Invitrogen Corp.) or FuGENE (Roche Diagnostics Corp.), with either wild-type MMP13 constructs containing C-terminal MycHis or Flag tags in pcDNA3.1 or a construct harboring the $\mathrm{SEMD}_{\mathrm{MO}}$ mutation that was introduced by the use of site-directed mutagenesis (QuikChange; Stratagene), as described previously (23). Forty-eight hours after transfection, the cells and supernatants were harvested. The cells were washed with PBS and lysates prepared by incubation in $0.5 \mathrm{ml}$ lysis buffer (20 mM Tris-HCl, pH 8, $137 \mathrm{mM} \mathrm{NaCl}, 10 \%$ glycerol, $1 \mathrm{mM} \mathrm{NaF}, 1 \mathrm{mM} \mathrm{Na} \mathrm{VO}_{4}, 0.1 \% \mathrm{NP}-40$ ) plus Complete Protease Inhibitor Cocktail (Roche Diagnostics Corp.) for 15 minutes on ice, followed by centrifugation at $14,000 \mathrm{~g}$ and $4^{\circ} \mathrm{C}$ for $10 \mathrm{~min}$ utes. The cell lysates were spun through a Microcon YM-10 Centrifugal Filter Unit with a cut-off $10 \mathrm{kDa}$ (Millipore), fractionated by $10 \%$ SDS-PAGE, and transferred to PVDF membranes (23). Western blot analyses were performed using antibodies against the tags or different MMP13 antibodies. The latter were as follows: a mouse monoclonal antibody (MAB181-15A12; EMD Biosciences) directed against the pro- and active forms of the human MMP13; a mouse monoclonal antibody (MAB 13424; Chemicon International) directed against the catalytic domain of human MMP13 (Figure 4$)$ that recognizes both latent $(\sim 55 \mathrm{kDa})$ and active ( $\sim 5 \mathrm{kDa}) \mathrm{MMP} 13$; a rabbit polyclonal antibody (AB19057; Chemicon International) directed against the pro-peptide region that recognizes the latent $(\sim 55 \mathrm{kDa}) \mathrm{MMP} 13$ precursor; and a rabbit polyclonal antibody (AB8114; Chemicon International) directed against the hinge region that recognizes latent $(\sim 55 \mathrm{kDa})$ and active $(\sim 45 \mathrm{kDa})$ MMP13. An appropriate secondary antibody, i.e., anti-mouse HRP (Bio-Rad Laboratories) was used at 1:3,000 and visualized with an enhanced chemiluminescence (ECL) kit (Amersham Biosciences) (23). IVT wild-type and mutant MMP13 were generated using the TnT Coupled Reticulocyte Lysate IVT System (Promega) as previously described (23).

Preparation of stable transfectants. Stable transfectants in the parent HEK293 cells were prepared after transfection of pcDNA3.1 alone (empty vector) or pcDNA3.1 encoding untagged wild-type or mutant F56S MMP13 cDNA. After transfection, the contents of each well from 6-well plates were transferred to $100-\mathrm{mm}$ diameter dishes and cultured in the presence of $400 \mu \mathrm{g} / \mathrm{ml} \mathrm{G} 418$ (Geneticin; Invitrogen Corp.). Approximately 10 neo-resistant clones/transfectants were identified and selected using the cylinder method, and cells were expanded to collect conditioned medium to use in proteolytic assays or for transient transfection with other
A

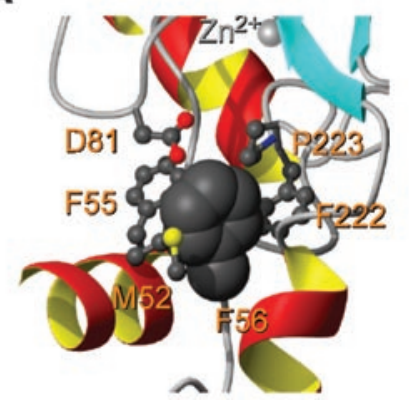

B

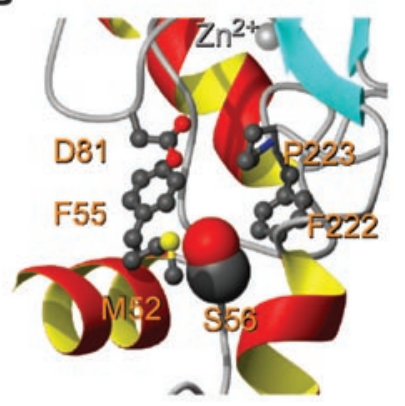

\section{Figure 9}

Proposed 3-dimensional model of the prodomain and catalytic domains of MMP13. (A) Wild-type MMP13. The F56 side chain lies buried in the interface between the prodomain (residues 20-103; Figure 4) and the catalytic domain (residues 104-270). (B) F56S MMP13. There is a more open structure with a cavity in this region. 
constructs in pcDNA3.1. For double transfection, the stable clones were transiently transfected as described above using pcDNA3.1 containing empty vector, wild-type Flag-tagged MMP13, or mutant F56S Flag-tagged MMP13. Transfected cells were then cultured for 48 hours with or without monensin, conditioned medium was harvested, cells were washed with PBS, and lysates were prepared by incubation as described above in $0.5 \mathrm{ml}$ lysis buffer. Conditioned medium and cell lysates were then analyzed by Western blotting with anti-Flag antibody.

Collagen proteolysis. Human proMMP13 cDNA in pcDNA 3.1 was stably transfected into HEK 293 cells that were then maintained in DMEM, $10 \%$ FCS. To obtain collagenase for these experiments, DMEM, 10\% FCS was removed and replaced with DMEM containing $0.2 \%$ lactalbumin, and after incubation for 48 hours, medium was harvested and concentrated 20-fold using the Centricon YM-30 Concentrator (Millipore). Collagenase digestion was carried out at $20^{\circ} \mathrm{C}$ in collagenase buffer (0.1 mM Tris- $\mathrm{HCl}$, pH 7.5, $5 \mathrm{mM} \mathrm{CaCl}_{2}, 0.1 \mathrm{M} \mathrm{NaCl}, 0.25 \mathrm{M}$ glucose) as previously described (47). Latent collagenase was activated by treatment with APMA, which was brought to a concentration of $1 \mathrm{mM}$ at the beginning of the incubation. For gelatinase assays, collagen was heated for 30 minutes at $90^{\circ} \mathrm{C}$ and cooled to $4^{\circ} \mathrm{C}$ until used. Incubation with the MMP13 preparation was performed for 15 hours at $20^{\circ} \mathrm{C}$ in a thermocycler. The reaction was stopped by the addition of EDTA to a final concentration of $50 \mathrm{mM}$. Samples were stored at $4^{\circ} \mathrm{C}$ prior to analysis by SDS-PAGE. Digestion products were resolved by SDS-PAGE using $7.5 \%$ acrylamide without reduction and gels were stained with GelCode Blue Stain Reagent (Pierce Biotechnology Inc.).

Three-dimensional modeling. A 3-dimensional model of the prodomain with the catalytic domain of MMP13 was derived using the structure of MMP13 catalytic domain alone (Protein Data Bank ID [PDB-ID] 830C; http://www.rcsb.org/pdb) and the structures of prodomain plus catalytic domain of proMMP2 (PDB-ID 1CK7) and proMMP3 (PDB-ID $1 \mathrm{SLM})$. We used the semiautomated modeling server SWISS-MODEL (http://expasy.org) (48). Briefly, the target sequence was threaded over the structure of the templates, built with ProMod II, and energy-minimized with Gromos 96 as described by Schwede et al. (48). The quality of the resulting model was verified automatically with WhatCheck (48) and manually with Deep View (http://expasy.org/spdbv). The interactions among the residues were derived with CSU and LPC software (http://bip.weizmann.ac.il/oca-bin/lpccsu) (49). Figure 9 was modeled with MOLMOL (http://hugin.ethz.ch/wuthrich/software/molmol/) and rendered with POV-Ray (http://www.povray.org).

\section{Acknowledgments}

We thank the Medical Research Council, UK (A.M. Kennedy, P.T. Christie, B. Harding, A.A.J. Pannett, A. Dearlove, C. Hartley, M.A. Nesbit, and R.V. Thakker), the Wellcome Trust (A.A.C. Reed and R.V. Thakker), the NIH (M. Inada and S.M. Krane), the European Union (C. López-Otín and L.M. Sánchez), and the Shriners Hospitals for Children, USA (M.P. Whyte) for support. We are grateful to William $\mathrm{H}$. McAlister for characterizing the radiographic findings.

Received for publication August 2, 2004, and accepted in revised form July 12, 2005.

Address correspondence to: Rajesh V. Thakker, Academic Endocrine Unit, Nuffield Department of Clinical Medicine, University of Oxford, Oxford Centre for Diabetes, Endocrinology and Metabolism (OCDEM), Churchill Hospital, Oxford OX3 7LJ, United Kingdom. Phone: 44-1865-857501; Fax: 44-1865-857502; E-mail: rajesh.thakker@ndm.ox.ac.uk.

Ann M. Kennedy and Masaki Inada contributed equally to this work.
1. Hall, C.M. 2002. International classification of nosology and classification of constitutional disorders of bone (2001). Am. J. Med. Genet. 113:65-77.

2. Superti-Furga, A., Bonafe L., and Rimoin D.L. 2001. Molecular-pathogenic classification of genetic disorders of the skeleton. Am. J. Med. Genet. 106:282-293.

3. Tiller, G.E., et al. 1995. Dominant mutations in the type II collagen gene, COL2A1, produce spondyloepimetaphyseal dysplasia, Strudwick type. Nat. Genet. 11:87-89.

4. ul Haque, M.F., et al. 1998. Mutations in orthologous genes in human spondyloepimetaphyseal dysplasia and the brachymorphic mouse. Nat. Genet. 20:157-162.

5. Patel, A.C., McAlister, W.H., and Whyte, M.P. 1993. Spondyloepimetaphyseal dysplasia: clinical and radiologic investigation of a large kindred manifesting autosomal dominant inheritance, and a review of the literature. Medicine (Baltimore). 72:326-342.

6. Whyte, M.P., Petersen, D.J., and McAlister, W.H. 1990. Hypotrichosis with spondylometaphyseal dysplasia in three generations: a new autosomal dominant syndrome. Am. J. Hum. Genet. 36:288-291.

7. Anderson, C.E., et al. 1982. Spondylometepiphyseal dysplasia, Strudwick type. Am. J. Med. Genet. 13:243-256.

8. Beighton, P., Gericke, G., Kozlowski, K., and Grobler, L. 1984. The manifestations and natural history of spondylo-epi-metaphyseal dysplasia with joint laxity. Clin. Genet. 26:308-317.

9. Sillence, D.O., Horton, W.A., and Rimoin, D.L. 1979. Morphological studies in the skeletal dysplasias. Am. J. Pathol. 96:813-870.
10. Borochowitz, Z.U., et al. 2004. Spondylo-epimetaphyseal dysplasia (SEMD) matrilin 3 type: homozygote matrilin 3 mutation in a novel form of SEMD. J. Med. Genet. 41:366-372.

11. Gertner, J.M., et al. 1997. Linkage studies of a Missouri kindred with autosomal dominant spondyloepimetaphyseal dysplasia (SEMD) indicate genetic heterogeneity. J. Bone Miner. Res. 12:1204-1209.

12. Lloyd, S.E., Pannett, A.A., Dixon, P.H., Whyte, M.P., and Thakker, R.V. 1999. Localization of familial benign hypercalcaemia, Oklahoma variant (FBHOk), to chromosome 19q13. Am. J. Hum. Genet. 64:189-195.

13. Nagase, H., and Woessner, J.F., Jr. 1999. Matrix metalloproteinases. J. Biol. Chem. 274:2191-2194.

14. Stamenkovic, I. 2003. Extracellular matrix remodelling: the role of matrix metalloproteinases. J. Pathol. 200:448-464.

15. Freije, J.M., et al. 1994. Molecular cloning and expression of collagenase-3, a novel human matrix metalloproteinase produced by breast carcinomas. J. Biol. Chem. 269:16766-16773.

16. Knauper, V., López-Otín, C., Smith, B., Knight, G., and Murphy, G. 1996. Biochemical characterization of human collagenase-3. J. Biol. Chem. 271:1544-1550.

17. Mitchell, P.G., et al. 1996. Cloning, expression, and type II collagenolytic activity of matrix metalloproteinase-13 from human osteoarthritic cartilage. J. Clin. Invest. 97:761-768.

18. Stahle-Backdahl, M., et al. 1997. Collagenase-3 (MMP-13) is expressed during human fetal ossification and re-expressed in postnatal bone remodeling and in rheumatoid arthritis. Lab. Invest. 76:717-728.
19. Pendas, A.M., Balbin, M., Llano, E., Jimenez, M.G., and Lopez-Otin, C. 1997. Structural analysis and promoter characterization of the human collagenase-3 gene (MMP13). Genomics. 40:222-233.

20. Eriksson, A.E., et al. 1992. Response of a protein structure to cavity-creating mutations and its relation to the hydrophobic effect. Science. 255:178-183.

21. Xu, B., et al. 2002. A cavity-forming mutation in insulin induces segmental unfolding of a surrounding alpha-helix. Protein. Sci. 11:104-116.

22. Hammond, C., and Helenius, A. 1995. Quality control in the secretory pathway. Curr. Opin. Cell Biol. 7:523-529.

23. Nesbit, M.A., et al. 2004. Characterisation of GATA3 mutations in the hypoparathyroidism, deafness and renal dysplasia (HDR) syndrome. J. Biol. Chem. 279:22624-22634.

24. Kopito, R.R., and Sitia, R. 2000. Aggresomes and Russell bodies. Symptoms of cellular indigestion? EMBO Rep. 3:225-231.

25. Goldberg, A., Cascio, P., Saric, T., and Rock, K. 2002. The importance of the proteasome and subsequent proteolytic steps in the generation of antigenic peptides. Mol. Immunol. 39:147-164.

26. Bergmann, U., Tuuttila, A., Stetler-Stevenson, W.G., and Tryggvason, K. 1995. Autolytic activation of recombinant human 72 kilodalton type IV collagenase. Biochemistry. 34:2819-2825.

27. Mizuta, R., Mizuta, M., Araki, S., and Kitamura, D. 2002. RAG2 is down-regulated by cytoplasmic sequestration and ubiquitin-dependent degradation. J. Biol. Chem. 277:41423-41427.

28. Teckman, J.H., et al. 2001. The proteasome participates in degradation of mutant alpha 1-antitrypsin $Z$ in the endoplasmic reticulum 
of hepatoma-derived hepatocytes. J. Biol. Chem. 276:44865-44872.

29. Dinter, A., and Berger, E.G. 1998. Golgi-disturbing agents. Histochem. Cell Biol. 109:571-590.

30. Morgunova, E., et al. 1999. Structure of human pro-matrix metalloproteinase-2: activation mechanism revealed. Science. 284:1667-1670.

31. Chung, L., et al. 2004. Collagenase unwinds triplehelical collagen prior to peptide bond hydrolysis EMBO J. 23:3020-3030.

32. Galazka, G., Windsor, L.J., Birkedal-Hansen, H., and Engler, J.A. 1996. APMA (4-aminophenylmercuric acetate) activation of stromelysin- 1 involves protein interactions in addition to those with cysteine-75 in the propeptide. Biochemistry. 35:11221-11227.

33. Goldring, M.B., et al. 1994 . Interleukin- $\beta$-modulated gene expression in immortalized human chondrocytes. J. Clin. Invest. 94:2307-2316.

34. Van Wart, H.E., and Birkedal-Hansen, H. 1990. The cysteine switch: a principle of regulation of metalloproteinase activity with potential applicability to the entire matrix metalloproteinase gene family. Proc. Natl. Acad. Sci. U. S. A. 87:5578-5582.

35. Gonzales, P.E., et al. 2004. Inhibition of the tumor necrosis factor- $\alpha$-converting enzyme by its pro domain. J. Biol. Chem. 279:31638-31645.

36. Gomis-Ruth, F.X. 2003. Structural aspects of the metzincin clan of metalloendopeptidases [review]. Mol. Biotechnol. 24:157-202.
37. Monzingo, A.F., and Matthews, B.W. 1984. Binding of $\mathrm{N}$-carboxymethyl dipeptide inhibitors to thermolysin determined by X-ray crystallography: a novel class of transition-state analogues for zinc peptidases. Biochemistry. 23:5724-5729.

38. Eriksson, A.E., Baase, W.A., and Matthews, B.W. 1993. Similar hydrophobic replacements of Leu99 and Phe 153 within the core of T4 lysozyme have different structural and thermodynamic consequences. J. Mol. Biol. 229:747-769.

39. Reboul, P., Pelletier, J.-P., Tardif, G., Cloutier, J.-M., and Martel-Pelletier, J. 1996. The new collagenase, collagenase- 3 is expressed and synthesized by human chondrocytes but not by synoviocytes. J. Clin. Invest. 97:2011-2019.

40. Inada, M., et al. 2004. Critical roles for collagenase-3 (Mmp13) in development of growth plate cartilage and in endochondral ossification. Proc. Natl. Acad. Sci.U.S. A. 101:17192-17197.

41. Stickens, D., et al. 2004. Altered endochondra bone development in matrix metalloproteinase 13-deficient mice. Development. 131:5883-5895.

42. Sires, U.I., et al. 1995. Complete degradation of type $\mathrm{X}$ collagen requires the combined action of interstitial collagenase and osteoclast-derived cathepsin-B. J. Clin. Invest. 95:2089-2095.

43. Fosang, A.J., Last, K., Knauper, V., Murphy, G., and Neame, P.J. 1996. Degradation of cartilage aggrecan by collagenase-3 (MMP-13). FEBS Lett. 380:17-20.
44. Martignetti, J.A., et al. 2001. Mutation of the matrix metalloproteinase 2 gene (MMP2) causes a multicentric osteolysis and arthritis syndrome. Nat. Genet. 28:261-265.

45. Gedeon, A.K., et al. 1999. Identification of the gene (SEDL) causing X-linked spondyloepiphyseal dysplasia tarda. Nat. Genet. 22:400-404.

46. Reid,E., Dearlove, A.M., Rhodes, M., and Rubinsztein, D.C. 1999. A new locus for autosomal dominant "pure" hereditary spastic paraplegia mapping to chromosome $12 \mathrm{q} 13$, and evidence for further genetic heterogeneity. Am. J. Hum. Genet. 65:757-763.

47. Jung, J.-C., et al. 2004. Studies on activity and expression of xenopus laevis matrix metalloproteinases: identification of a novel role for the hormone prolactin in regulating collagenolysis in both amphibians and mammals. J. Cell. Physiol. 201:155-164.

48. Schwede, T., Kopp, J., Guex, N., and Peitsch, M.C. 2003. SWISS-MODEL: an automated protein homology-modeling server. Nucleic Acids Res. 31:3381-3385.

49. Sobolev, V., Sorokine, A., Prilusky, J., Abola, E.E., and Edelman, M. 1999. Automated analysis of interatomic contacts in proteins. Bioinformatics. 15:327-332.

50. Gyapay, G., et al. 1994. The 1993-94 Genethon human genetic linkage map. Nat. Genet. 7:246-339. 